\title{
A novel vaporisation model for a single-component drop in high temperature air streams
}

\author{
Simona Tonini*, Gianpietro Elvio Cossali \\ Engineering Department, Università degli studi di Bergamo, Viale Marconi 5, 24044 Dalmine, BG, Italy
}

\section{A R T I C L E I N F O}

\section{Article history:}

Received 18 April 2013

Received in revised form

19 July 2013

Accepted 7 August 2013

Available online

\section{Keywords:}

Drop evaporation

Film theory

Analytical model

\begin{abstract}
A B S T R A C T
A new model for drop evaporation has been developed, including the effect of gas convection according to the film theory, by relating the thermal and diffusional film thicknesses to the gas stream Reynolds number. The model solves the species, momentum and energy conservation equations in a radial coordinate system, accounting explicitly for the gas density dependence on temperature and vapour concentration. The model has been tested under a wide range of gas temperatures, Reynolds numbers and liquid species and the results have been compared against experimental data available in the scientific literature and with the predictions from the classical extended film model. The present model, together with relieving some of the physical inconsistencies of the classical models, which become not negligible at high gas temperature, also shows a rather good agreement with the experimental data for the selected operating conditions.
\end{abstract}

(c) 2013 Elsevier Masson SAS. All rights reserved.

\section{Introduction}

The modelling of spherical drops evaporating in a gaseous environment has wide engineering applications like in spray combustion, spray cooling, spray drying, fire suppression, etc. [1]. Drop evaporation is a complex two-phase flow phenomenon, which modelling should take into account the effect of transient liquid heating, gas phase convection and variable physical properties [2].

After the formulation of the classical " $D^{2}$ law" [3,4], which predicts that the square of droplet diameter decreases linearly with time, many remarkable advances in the modelling of drop evaporation have been reported, including the effect of variable thermophysical properties, non-unity Lewis number in the gas film surrounding the drop, transient liquid heating, gas phase convection, non-equilibrium evaporation law assumption (refer to [2,57], for recent reviews on drop evaporation modelling addressing the previous issues).

Among the variety of drop evaporation models available in the scientific literature, the extended film model of Abramzon and Sirignano [8], developed more than 20 years ago, remains the most commonly used model for spray calculations, due to its efficient implementation in CFD codes. This model is based on the one-

\footnotetext{
* Corresponding author.

E-mail address: simona.tonini@unibg.it (S. Tonini).
}

dimensional "stagnant film theory", incorporating the Stefan flow effect on the thicknesses of the thermal and diffusional films.

Heat and mass transfers of an evaporating drop in a convective environment are usually expressed introducing correlations to Nusselt and Sherwood numbers. Different empirical correlations are available in the scientific literature (refer to [1,2,9] for review) and have been implemented either in single drop or spray calculations. Renksizbulut and Yuen [9] proposed a correlation for the Nusselt number valid for single component drops evaporating in high-temperature air streams, which at low-temperature environments reduces to the standard heat transfer equation of Ranz and Marshall [10,11].

The drop evaporation modelling proposed in the open literature usually refers to a limited number of classical experimental data bases, although only few of them report all the necessary information to correctly reproduce the test cases. Ranz and Marshall [11], back in 1952, proposed a comprehensive study on water and benzene evaporation at quasi steady-state conditions, reporting the evaporation rate and drop temperatures at low and moderate evaporating conditions. Few years later Downing [12] measured the quasi steady-state temperature, which is usually approximated as the wet-bulb temperature, for benzene drops and the evaporation history of hexane drops in air streams at different temperatures, while Eisenklam et al. [13] measured the quasi-steady evaporation rates of different liquids in convective high-temperature air environment. The transient evaporation of a decane millimeter drop at high temperature air stream conditions has been recorded by Wong 


\begin{tabular}{|c|c|c|c|}
\hline \multicolumn{2}{|c|}{ Nomenclature } & $\begin{array}{l}U \\
\dot{V}_{\mathrm{ev}} \\
Y\end{array}$ & $\begin{array}{l}\text { velocity, } \mathrm{m} / \mathrm{s} \\
\text { volumetric evaporation rate, } \mathrm{m}^{3} / \mathrm{s} \\
\text { non-dimensional evaporation rate }\end{array}$ \\
\hline \multicolumn{4}{|c|}{ Roman symbols } \\
\hline \multicolumn{2}{|c|}{$A, B, F, G$ non-dimensional functions } & \multicolumn{2}{|c|}{ Greek symbols } \\
\hline$B_{\mathrm{f}}$ & Spalding heat transfer number & \multicolumn{2}{|c|}{$\Lambda, \theta, \Gamma, \Psi$ non-dimensional parameters } \\
\hline$B_{\mathrm{M}}$ & Spalding mass transfer number & $\rho$ & density, $\mathrm{kg} / \mathrm{m}^{3}$ \\
\hline$B_{\mathrm{M}}^{*}$ & corrected Spalding mass transfer number & $\rho_{j}$ & mass concentration of species $j, \mathrm{~kg} / \mathrm{m}^{3}$ \\
\hline$B_{\mathrm{T}}$ & Spalding heat transfer number & $\chi$ & mass fraction \\
\hline$c$ & specific heat (at constant pressure), $\mathrm{J} / \mathrm{kg} \mathrm{K}$ & $\mu$ & dynamic viscosity, $\mathrm{kg} / \mathrm{ms}$ \\
\hline$D$ & drop diameter, $\mathrm{m}$ & $\zeta$ & non-dimensional coordinate \\
\hline \multicolumn{4}{|c|}{ diffusion coefficient of vapour in air, $\mathrm{m}^{2} / \mathrm{s}$} \\
\hline$h_{\mathrm{LV}}$ & specific heat of vaporization, $\mathrm{J} / \mathrm{kg}$ & \multicolumn{2}{|c|}{ Subscripts } \\
\hline$k$ & thermal conductivity, $\mathrm{W} / \mathrm{mK}$ & $\mathrm{cr}$ & critical \\
\hline Le & Lewis number & $\mathrm{f}$ & film conditions \\
\hline $\mathrm{Nu}$ & Nusselt number & $\mathrm{g}$ & gas \\
\hline & evaporation rate, $\mathrm{kg} / \mathrm{s}$ & 1 & liquid \\
\hline $\mathrm{Mm}$ & molar mass, $\mathrm{kg} / \mathrm{kmol}$ & rad & radiation \\
\hline$P$ & pressure, Pa & s & drop surface \\
\hline$P_{\mathrm{v}}$ & vapour partial pressure, $\mathrm{Pa}$ & $\mathrm{T}$ & total \\
\hline$P_{v s}$ & saturation vapour pressure, $\mathrm{Pa}$ & $\mathrm{v}$ & vapour \\
\hline $\operatorname{Pr}$ & Prandtl number & 0 & initial conditions \\
\hline$\dot{Q}$ & heat rate, $\mathrm{W}$ & $\infty$ & infinity \\
\hline$r$ & radial coordinate, $\mathrm{m}$ & & \\
\hline$R$ & universal gas constant, $\mathrm{J} / \mathrm{kmol} \mathrm{K}$ & \multicolumn{2}{|c|}{ Superscripts } \\
\hline Re & Reynolds number & $A-S$ & Abramzon and Sirignano \\
\hline$R_{0}$ & drop radius, $m$ & calc & calculated \\
\hline$R_{2}$ & thermal film outer radius, $\mathrm{m}$ & $\exp$ & experimental \\
\hline$R_{3}$ & diffusional film outer radius, $\mathrm{m}$ & $\sim$ & non-dimensional \\
\hline Sc & Schmidt number & & \\
\hline Sh & Sherwood number & \multicolumn{2}{|c|}{ Abbreviation } \\
\hline$t$ & time, $s$ & CFD & computational fluid dynamics \\
\hline$T$ & temperature, $\mathrm{K}$ & LHS & left hand side \\
\hline$u$ & specific internal energy, $\mathrm{J} / \mathrm{kg}$ & RHS & right hand side \\
\hline
\end{tabular}

and Lin in Ref. [14] and their data represent a common reference for model validation.

As previously mentioned, these four data bases of experimental measurements on single-component drop evaporation contain all the requested information to be compared with the corresponding model predictions and they have been selected for the purposes of the present investigation. Other works reporting experiments on single-component drop vaporisation in low and high temperature air streams can be found in the scientific literature (see Refs. $[15,16,9])$ and they have been referred for model comparison (see Ref. [8] for example), but they either refer to previously published measurements or they do not specify all the operating conditions to reproduce the selected test-cases, and for these reasons they were not used in the present study.

The target of this work is to extend the model recently developed by the authors [17], which takes into account gas temperature and density gradient effects, including the contribution of convection to liquid drop evaporation, with the main scope to efficiently implement it in comprehensive spray simulations. The new model relieves some of the inconsistencies of previous drop evaporation models, achieving a good accuracy in the predictions particularly at high evaporation rate conditions, typical of high temperature, high Reynolds number environments.

The description of the mathematical model and the derivation of the analytical solution are presented in the following section, followed by the discussion on the comparison between the model results, as predicted by the present model and by the classical extended film model of Abramzon and Sirignano [8], and a wide range of experimental data on quasi steady-state and transient drop evaporation selected from the scientific literature. Finally the main conclusions are briefly summarised.

\section{Mathematical model}

The proposed model for spherical drop evaporation in hot gaseous environment under convective conditions is developed following a method similar to that proposed by Sirignano and Abramzon [8], based on film theory.

The following species, momentum and energy time independent conservation equations (see Ref. [17] for a discussion on the assumed simplifications):

$$
\begin{aligned}
& \frac{\mathrm{d}}{\mathrm{d} r}\left(r^{2} \rho_{\mathrm{v}} U-r^{2} D_{\mathrm{v}} \rho \frac{\mathrm{d} \chi_{\mathrm{v}}}{\mathrm{d} r}\right)=0 \\
& \frac{\mathrm{d}}{\mathrm{d} r}\left(r^{2} \rho_{\mathrm{g}} U-r^{2} D_{\mathrm{v}} \rho \frac{\mathrm{d} \chi_{\mathrm{g}}}{\mathrm{d} r}\right)=0 \\
& \rho U \frac{\mathrm{d} U}{\mathrm{~d} r}=-\frac{\mathrm{d} P_{\mathrm{T}}}{\mathrm{d} r}+\mu\left(\frac{\mathrm{d}^{2} U}{\mathrm{~d} r^{2}}+\frac{2}{r} \frac{\mathrm{d} U}{\mathrm{~d} r}\right) \\
& \rho U c \frac{\mathrm{d} T}{\mathrm{~d} r}=k\left(\frac{\mathrm{d}^{2} T}{\mathrm{~d} r^{2}}+\frac{2}{r} \frac{\mathrm{d} T}{\mathrm{~d} r}\right)
\end{aligned}
$$


where $\rho=\rho_{\mathrm{v}}+\rho_{\mathrm{g}}$ is the gas mixture density, $\chi_{j}=\rho_{j} / \rho$ the specie mass fraction, are solved on a finite shell around the droplet, accounting explicitly for the dependence of the gas density on temperature and species concentration.

Two different partially overlapping regions are defined for the solution of the heat and mass transfer problems, defined by a thermal $\left(R_{2}-R_{0}\right)$ and a diffusional $\left(R_{3}-R_{0}\right)$ thickness, where $R_{0}$ is the drop radius.

\subsection{Heat rate}

The energy Equation (3) can be solved independently. Introducing the non-dimensional variables: $\tilde{T}=T / T_{\infty} ; \zeta=R_{0} / r ; Y=\dot{m}_{\mathrm{ev}} /\left(4 \Pi R_{0} D_{\mathrm{v}} \rho_{\infty} L e\right), \dot{m}_{\mathrm{ev}}$ the vaporisation rate and $L e$ the Lewis number, and imposing the B.C.:

$T\left(\zeta=\zeta_{2}\right)=T_{\infty} ; T(\zeta=1)=T_{\mathrm{S}}$

where $\zeta_{2}=R_{2} / R_{0}$, the analytical solution can be presented as:

$\tilde{T}=\left(1-\tilde{T}_{\mathrm{s}}\right) \frac{e^{-Y \zeta}-e^{-Y}}{e^{-Y \zeta_{2}}-e^{-Y}}+\tilde{T}_{\mathrm{s}}$

obtained by integrating Equation (3), under the assumption that the specific heat $\mathrm{c}$ and the thermal conductivity $k$ are constant. Their values are assumed to be calculated at reference film conditions, which change with liquid and gas temperatures and vapour concentration.

The sensible heat rate exchanged by the drop with the environment, defined as usual as:

$\dot{Q}=-k\left(\frac{\partial T}{\partial r}\right)_{r=R_{0}} 4 \pi R_{0}^{2}$

can then be evaluated in non-dimensional form as:

$\tilde{Q}=\frac{\dot{Q}}{4 \pi R_{0} k T_{\infty}}=\left(\tilde{T}_{\mathrm{s}}-1\right) \frac{Y e^{-Y}}{e^{-Y \zeta_{2}}-e^{-Y}}$

\subsection{Evaporation rate}

Following [17] the non-dimensional variables:

$G=\ln \left(\chi_{\mathrm{g}}\right) ; \quad \tilde{U}=\frac{U 4 \pi R_{0}^{2} \rho_{\infty}}{\dot{m}_{\mathrm{ev}}} ; \tilde{\rho}=\frac{\rho}{\rho_{\infty}}$

are introduced and, after noticing that Equation (1a) and (1b) yield:

$\tilde{U}=-\frac{\zeta^{2}}{\hat{m}_{\mathrm{ev}}} G_{\zeta}$

$\tilde{\rho}=-\frac{\widehat{m}_{\mathrm{ev}}}{G_{\zeta}}$

Equation (2) transforms to:

$$
\begin{aligned}
& \frac{\zeta^{2} G_{\zeta}^{2}}{\left[\zeta^{4} G_{\zeta}^{2}-\Lambda\left(1+\theta e^{G}\right) \tilde{T}\right] \frac{\widehat{m}_{\mathrm{ev}}}{S c}} \frac{\mathrm{d}^{2} \zeta^{2} G_{\zeta}}{\mathrm{d} \zeta^{2}} \\
& =-G_{\zeta \zeta}+G_{\zeta} \frac{\Lambda\left(G_{\zeta} \theta e^{G} \tilde{T}+\left(1+\theta e^{G}\right) \tilde{T}_{\zeta}\right)+2 \zeta^{3} G_{\zeta}^{2}}{\Lambda\left(1+\theta e^{G}\right) \tilde{T}-\zeta^{4} G_{\zeta}^{2}}
\end{aligned}
$$

where $\Lambda=\left(R T_{\infty} R_{0}^{2}\right) /\left(M m_{\mathrm{v}} D_{\mathrm{v}}^{2}\right)$ and $\theta=\left(\mathrm{Mm}_{\mathrm{v}}-\mathrm{Mm}_{\mathrm{g}}\right) / \mathrm{Mm}_{\mathrm{g}}$. Note that the LHS has been corrected from the formulation reported in Ref. [17], where Le appeared instead of the Schmidt number, Sc.

The boundary conditions are set by imposing the value of $\chi_{\mathrm{v}}$ at the film edge $\left(\zeta=\zeta_{3}\right)$ and the value of the vapour pressure $P_{\mathrm{v}}$ on the drop surface $(\zeta=1)$, yielding:

$G\left(\zeta_{3}\right)=\ln \left(\chi_{\mathrm{g}, \infty}\right)$

$G_{\zeta}(1)=-\frac{\widehat{m}_{\mathrm{ev}} \tilde{T}_{\mathrm{s}}}{\widehat{P}_{\mathrm{vs}}}\left(1-e^{G(1)}\right)$

where $\widehat{P}_{\mathrm{vs}}=P_{\mathrm{vs}} \mathrm{Mm}_{\mathrm{v}} / R T_{\infty} \rho_{\infty}=\chi_{\mathrm{vs}} \tilde{\rho}_{\mathrm{s}} \tilde{T}_{\mathrm{s}}$.

Moreover, the condition on the values of the mixture density $\rho$ at the film edge $\left(\rho\left(\zeta_{3}\right)=\rho_{\infty}\right)$ yields (see Equation $\left.(6)\right)$ :

$G_{\zeta}\left(\zeta_{3}\right)=-\widehat{m}_{\mathrm{ev}}$

It has been shown [17] that in a large variety of conditions of practical interest the parameter $\Lambda$ assumes quite large values, then the asymptotic condition $\Lambda \rightarrow \infty$ yields the following simplified form of Equation (7):

$G_{\zeta \zeta}=G_{\zeta}\left[\frac{\theta e^{G} G_{\zeta}}{\left(1+\theta e^{G}\right)}+\frac{\tilde{T}_{\zeta}}{\tilde{T}}\right]$

that admits the analytical solution:

$G(\zeta)=\ln \left(\frac{e^{A F(\zeta)+B}}{1-\theta e^{A F(\zeta)+B}}\right)$

where:

$F(\zeta)=\int \tilde{T} \mathrm{~d} \zeta$

$A=\frac{\ln \frac{K_{\mathrm{s}}}{K_{\infty}}}{F(1)-F\left(\zeta_{3}\right)} ; B=\frac{F(1) \ln K_{\infty}-F\left(\zeta_{3}\right) \ln K_{\mathrm{s}}}{F(1)-F\left(\zeta_{3}\right)} ;$

$K_{\mathrm{s}}=\frac{1-\chi_{\mathrm{v}, \mathrm{s}}}{1+\theta\left(1-\chi_{\mathrm{v}, \mathrm{s}}\right)} ; \quad K_{\infty}=\frac{1-\chi_{\mathrm{v}, \infty}}{1+\theta\left(1-\widehat{P}_{\mathrm{v}, \infty}\right)}$

which is similar to the solution already reported in Ref. [17] (valid for infinite film thickness), to notice that the definition of $K_{\mathrm{s}}$ is corrected by the misprinting appearing in that work. The application of the B.C. (8a), (8b) and (9) yields the following implicit form for the non-dimensional evaporating mass flow rate:

$$
\begin{aligned}
Y & +\left(\tilde{T}_{\mathrm{S}}-1\right)\left(Y \frac{e^{-Y \zeta_{2}}}{\left(e^{-Y \zeta_{2}}-e^{-Y}\right)}-\frac{e^{-Y \zeta_{3}}-e^{-Y}}{\left(e^{-\zeta_{2} Y}-e^{-Y}\right)\left(1-\zeta_{3}\right)}\right) \\
& =\frac{\Gamma}{1-\zeta_{3}} \ln \left(1+B_{\mathrm{M}}^{*}\right)
\end{aligned}
$$

where $\quad \Gamma=\widehat{P}_{\mathrm{v}, \mathrm{cr}} / \mathrm{Le}, \quad \widehat{P}_{\mathrm{v}, \mathrm{cr}}=1+\theta\left(1-\chi_{\mathrm{v}, \infty}\right)$, $B_{\mathrm{M}}^{*}=\left(\widehat{P}_{\mathrm{vs}}-\chi_{\mathrm{v}, \infty}\right) /\left(\widehat{P}_{\mathrm{v}, \mathrm{cr}}-\widehat{P}_{\mathrm{v}, \mathrm{s}}\right)$.

The form of Equation (11) suggests a similarity with the model of Abramzon and Sirignano [8] (see following Equation (17)) where the Spalding mass transfer number $B_{\mathrm{M}}$ is here substituted by the parameter $B_{\mathrm{M}}^{*}$. 


\subsection{Model assumptions}

The solutions (5) and (11) for sensible heat and evaporation rates require the definition of the thermal $\left(R_{2}-R_{0}\right)$ and diffusional $\left(R_{3}-R_{0}\right)$ film thicknesses, or equivalently the definition of $\zeta_{2}=R_{0} /$ $R_{2}$ and $\zeta_{3}=R_{0} / R_{3}$, respectively. Since the sensible heat rate in nondimensional form (5) can be written by introducing the Nusselt number $\left(\mathrm{Nu}^{*}\right)$ as:

$\tilde{Q}=\frac{N u^{*}}{2}\left(\tilde{T}_{\mathrm{s}}-1\right)$

a comparison with Equation (5) yields promptly the following relation between $\zeta_{2}$ and $N u^{*}$ :

$\zeta_{2}=1-\frac{1}{Y} \ln \left(1+\frac{2 Y}{N u^{*}}\right)$

The present model assumes now that $\mathrm{Nu}^{*}$ can be evaluated by the following empirical correlation proposed by Renksizbulut and Yuen [9], valid for evaporating droplets in hot environment:

$N u^{*}=\frac{2+0.6 \operatorname{Re}_{\mathrm{f}}^{1 / 2} \operatorname{Pr}_{\mathrm{f}}^{1 / 3}}{1+B_{\mathrm{f}}}$

with the Spalding heat transfer number $B_{\mathrm{f}}$, at film conditions, calculated as follows [4]:

$B_{\mathrm{f}}=\frac{c_{\mathrm{f}}\left(T_{\infty}-T_{\mathrm{s}}\right)}{h_{\mathrm{LV}}}$

where $h_{\mathrm{LV}}$ is the latent heat of vaporisation, calculated at the drop temperature, and $c_{\mathrm{f}}$ the gaseous heat capacity at film conditions defined according to the reference state method:

$T_{\mathrm{f}}=T_{\mathrm{s}}+\alpha_{\mathrm{f}}\left(T_{\infty}-T_{\mathrm{s}}\right)$

$\chi_{\mathrm{f}}=\chi_{\mathrm{s}}+\alpha_{\mathrm{f}}\left(\chi_{\infty}-\chi_{\mathrm{s}}\right)$

where $\alpha_{\mathrm{f}}$ is assumed to be equal to $1 / 2$, following [9]. To notice that $\alpha_{\mathrm{f}}=1 / 3$ (" $1 / 3$ rd rule") is suggested by Ref. [15] and used in Ref. [8].

The diffusional film thickness (or equivalently $\zeta_{3}$ ) is instead estimated from an analogy with the model of Abramzon and Sirignano [8], which is largely used to account for the convection effects on droplet evaporation without assuming explicit dependence of gas density on temperature and specie concentrations. The model, also based on the film theory approach [18], yields the following simple relation to account for convection when evaluating the evaporation rate from a spherical drop in gaseous environment:

$Y^{\mathrm{A}-\mathrm{S}}=\frac{S h^{\mathrm{A}-\mathrm{S}}}{2 L e} \ln \left(1+B_{\mathrm{M}}\right)$

where:

$B_{\mathrm{M}}=\frac{\chi_{\mathrm{v}, \mathrm{s}}-\chi_{v, \infty}}{1-\chi_{\mathrm{v}, \mathrm{s}}} ; S h^{\mathrm{A}-\mathrm{S}}=2+\frac{\left(S h_{0}-2\right)}{F_{\mathrm{M}}\left(B_{\mathrm{M}}\right)}$

$S h_{0}$ is the Sherwood number calculated from a classical correlation [8]:

$S h_{0}=2+0.552 R e_{\mathrm{f}}^{1 / 2} S c_{\mathrm{f}}^{1 / 3}$

and $F_{\mathrm{M}}$ is an empirical relation suggested in Ref. [8]:
$F_{\mathrm{M}}\left(B_{\mathrm{M}}\right)=\left(1+B_{\mathrm{M}}\right)^{0.7} \frac{\ln \left(1+B_{\mathrm{M}}\right)}{B_{\mathrm{M}}}$

According to [8], Equation (19) overestimates the evaporation rate at low Reynolds numbers ( $\operatorname{Re} \leq 10$ ), resulting to be too sensitive to small velocity fluctuations. Alternative expressions have been suggested and can be found in the literature (see Ref. [19] for reference) and can be easily implemented in the present model.

The Abramzon and Sirignano model [8] is based on Fuchs [20] solution of Equation (1(a-b)) assuming constant gas density and $\operatorname{Re}=0$, and in fact Equation (17) transforms to the Fuchs solution:

$Y_{R e=0}^{\mathrm{A}-\mathrm{S}}=\frac{1}{L e} \ln \left(1+B_{\mathrm{M}}\right)$

when $S h_{0}=2$. Convection effects are then introduced by multiplying the RHS by $S h / 2$.

Similarly, Equation (11) transforms to the solution given in [17]:

$Y_{R e=0}+\left(\tilde{T}_{\mathrm{S}}-1\right)\left(\frac{Y_{R e=0}}{1-e^{-Y_{R e=0}}}-1\right)=\Gamma \ln \left(1+B_{\mathrm{M}}^{*}\right)$

when the drop is assumed to evaporate under non-convective conditions $(\operatorname{Re}=0)$, i.e. for $\zeta_{3}=0$.

In an analogous way, the RHS of Equation (11) can be written under the form $\left(S h^{*} / 2\right) \Gamma \ln \left(1+B_{M}^{*}\right)$, yielding a simple relation for $\zeta_{3}$ :

$\zeta_{3}=1-\frac{2}{S h^{*}}$

assuming again as in Ref. [8] that:

$S h^{*}=2+\frac{\left(S h_{0}-2\right)}{F_{\mathrm{M}}\left(B_{\mathrm{M}}^{*}\right)}$

where, on the basis of the same similarity, the Spalding mass transfer number $B_{\mathrm{M}}$ is now substituted by $B_{\mathrm{M}}^{*}$.

This yields the final relation for the non-dimensional evaporation rate:

$Y+\left(\tilde{T}_{\mathrm{S}}-1\right) \frac{N u^{*}}{2}\left[1+\frac{2 Y}{N u^{*}}-\frac{S h^{*}}{2 Y}\left(e^{\frac{2 Y}{S h^{*}}}-1\right)\right]=\frac{S h^{*}}{2} \Gamma \ln \left(1+B_{\mathrm{M}}^{*}\right)$

where Equation (13) was also used. It should be noticed that when $R e \rightarrow 0$, both $\mathrm{Nu}^{*}$ and $\mathrm{Sh}^{*} \rightarrow 2$, and Equation (23) transforms to (21), proposed in Ref. [17] for the evaporation of spherical drops in absence of convection.

Equations (12) and (23) represent the proposed new model for evaluating sensible heat and evaporation rates from a spherical drop in hot convective environment, accounting for the explicit and important dependence of the gas density on both temperature and specie concentration.

\subsection{Drop asymptotic temperature}

The majority of experimental measurements on quasi steadystate drop evaporation, available in the scientific literature, assumes that the liquid drop has already reached its asymptotic temperature, which depends on the operating conditions, and transient liquid heating may be neglected. In the present model the drop asymptotic temperature $T_{\mathrm{s}}$ is calculated solving Equation (11) in combination with the energy conservation equation under steady-state conditions:

$\dot{m}_{\mathrm{ev}} h_{\mathrm{LV}}=2 \pi R_{0} k_{\mathrm{f}} N u\left(T_{\infty}-T_{\mathrm{s}}\right)+\dot{Q}_{\mathrm{rad}}$ 
being $k_{\mathrm{f}}$ the gas phase thermal conductivity at reference film condition (see Equations (15) and (16)) and $\dot{Q}_{\text {rad }}$ the radiation heat rate. The modelling of thermal radiation has been omitted in the present contribution since the focus is on the prediction of the evaporation rate. Thermal radiation effect may play a not negligible role for drops evaporating under high gas temperature conditions, as stated in Ref. [21], and they can be taken into account with a suitable model calculating the term $\dot{Q}_{\text {rad }}$ in Equation (24).

The Nusselt number appearing in Equation (24) is calculated from Equation (14) when the present model is implemented, while the following expression is used for the Abramzon and Sirignano model, according to [8]:

$N u^{\mathrm{A}-\mathrm{S}}=2+\frac{\left(N u_{0}-2\right)}{F_{\mathrm{T}}}$

where $N u_{0}$ is the Nusselt number, calculated as follows according to [8] (refer to the above comment for $S h_{0}$, Equation (19), about the sensitivity of $\mathrm{Nu}_{0}$ to small velocity fluctuation at low Reynolds number):

$N u_{0}=2+0.552 R e_{\mathrm{f}}^{1 / 2} \operatorname{Pr}_{\mathrm{f}}^{1 / 3}$

and the coefficient $F_{\mathrm{T}}$ is empirically evaluated as:

$F_{\mathrm{T}}=\left(1+B_{\mathrm{T}}\right) \frac{0.7}{\ln \left(1+B_{\mathrm{T}}\right)} B_{\mathrm{T}}$

$B_{\mathrm{T}}=\left(1+B_{\mathrm{M}}\right)^{\phi}-1$

$\phi=\frac{c_{\mathrm{f}}}{c_{\infty}} \frac{S h^{\mathrm{A}-\mathrm{S}}}{N u^{\mathrm{A}-\mathrm{S}}} \frac{1}{L e}$

Note that the drop temperature is assumed uniform in the present investigation, according to the infinite conductivity model [8], and no detailed modelling of thermal distribution inside the drop has been included, either for the steady-state and transient calculations performed, which results are presented and commented in the next section. This assumption is justified in view of the main target of this study that is the formulation of a novel model of drop evaporation under high vaporisation rate conditions. Additional complexities related to this phenomenon are then neglected in the present work.

\subsection{Step-by-step numerical procedure}

The model has been implemented to calculate the drop temperature and evaporation rate, at quasi steady-state conditions or at each time step in case of a transient test-case. The implementation requires an iterative procedure, which is schematically summarised as follows:

1. The drop $\left(T_{\mathrm{s}}, R_{0}, \mathrm{Mm}_{\mathrm{v}}\right)$ and gas $\left(T_{\infty}, P_{\infty}, R e, \chi_{\mathrm{v}, \infty}\right)$ characteristics are given as input.

2. The vapour concentration at drop surface is calculated, $\chi_{\mathrm{v}, \mathrm{s}}=\chi_{\mathrm{v}, \mathrm{s}}\left(T_{\mathrm{s}}, P_{\infty}, \mathrm{Mm}_{\mathrm{v}}\right)$.

3. The liquid and gaseous physical properties, both in the gas film and in the undisturbed region far from the drop, are updated (refer to Equation (15) and (16) for gas properties at film conditions).

4. Calculation of Nusselt and Sherwood numbers from Equations (14) and (22), respectively.

5. Solution of the implicit Equation (23) for the calculation of the evaporation rate. This step requires an internal iterative procedure.
Table 1

Operating conditions.

\begin{tabular}{|c|c|c|c|c|}
\hline Reference & Species & $D_{0}(\mathrm{~mm})$ & $T_{\infty}(\mathrm{K})$ & $\operatorname{Re}$ \\
\hline $\begin{array}{l}\text { Ranz and Marshall, } \\
1952[11]\end{array}$ & Water, benzene & $0.954,1.1$ & $290-356$ & $2-220$ \\
\hline Downing, 1966 [12] & Hexane, benzene & 1.648 & $437-546$ & $48-66$ \\
\hline $\begin{array}{l}\text { Eisenklam et al., } \\
1967[13]\end{array}$ & $\begin{array}{l}\text { Heptane, pentane, } \\
\text { methyl alcohol, ethyl } \\
\text { alcohol, water, benzene }\end{array}$ & $0.025-2$ & $473-873$ & $0.02-20$ \\
\hline $\begin{array}{c}\text { Wong and Lin, } \\
1992[14]\end{array}$ & Decane & 1.961 & 1000 & 17 \\
\hline
\end{tabular}

6. Solution of the Equation (24) for the calculation of the drop temperature $T_{\mathrm{s}}$. Under transient conditions the left hand side of Equation (24) should be replace by the transient term $m_{\mathrm{d}} c_{l} \mathrm{~d} T_{\mathrm{s}} /$ $\mathrm{d} t$, where $c_{1}$ is the specific liquid capacity, to calculate the new drop temperature, while the new drop size is calculated from mass conservation equation, once the evaporation rate is known.

The steps 2/6 are repeated until a convergence criteria in the calculation of asymptotic drop temperature $T_{\mathrm{s}}$ is satisfied.

\section{Comparison with experimental data}

The model has been implemented to predict the heat and mass transfer rates of liquid drops under quasi steady-state and transient conditions and the results are compared with four data bases of experimental measurements reported in Refs. [11-14], which have been selected among the classical ones available in the scientific literature since they contain all the information on the operating conditions and experimental results requested to correctly reproduce the test cases. The selected data bases are commonly used by model developers for validation against well-established experimental data.

Table 1 summarises the operating conditions investigated, accounting for the vaporisation of eight species of liquid drops, flowing in hot air at temperature ranging from $290 \mathrm{~K}$ up to $1000 \mathrm{~K}$ and Reynolds number from 0.02 up to 220 .

The present model and the extended film model of Abramzon and Sirignano (A-S '89 model [8],), which is usually implemented in comprehensive spray simulations due to its good compromise between model accuracy and computational requests, have been compared with the experimental data reported in the four data sets, as described in the following sections.

For all the test case conditions the drop Biot number has been calculated. The results confirm that the Biot number is always lower than 1 , with a maximum value equal to 0.16 estimated for ethanol drops at higher gas temperature conditions. This justifies the assumption of uniform temperature in the liquid phase. Note that the infinite conductivity model is implemented in the present analysis when comparison with time dependent data is needed, without accounting for the effect of internal liquid circulation by means of an "effective" liquid conductivity (see Ref. [8] for reference), which would further reduce the Biot number, making this assumption even more reliable.

As stated in the scientific literature [6], in case of very small liquid drops (in the range of sub-micrometers) or under high gas pressure environments (well above $1 \mathrm{~atm}$ ) the properties of the gas in the vicinity of the liquid-gas interface can depend not only on the average velocities of molecules, but also on the distribution of molecules by velocities. Consequently the modelling of drop evaporation should take into account these kinetic effects (see Refs. $[6,22]$, for reference). All the test cases studied in the present investigation assume that the drop size is of the order of magnitude 
that makes kinetic effect reasonably negligible. Moreover, the calculations of the test cases analysing the transient evolution of drop size have been stopped when the drop diameter has been reduced down to $1 / 10$ of the initial size, which is still above the range of drop sizes where kinetic effect may play a role, confirming that the assumption made by the model is justifiable.

\subsection{Data base of Ranz and Marshall, 1952 [11]}

The first data base of experimental measurements is from Ref. [11], which reports the quasi steady-state volumetric evaporation rate $\left(\dot{V}_{\mathrm{ev}}\right)$ for water and benzene drops under moderately low evaporating conditions. The liquid temperature ranges between $278.6 \mathrm{~K}$ up to $301 \mathrm{~K}$ for water drops and between $270.9 \mathrm{~K}$ and $276.7 \mathrm{~K}$ for benzene drops. The drop size was measured with a tolerance of $\pm 0.3 \mathrm{~mm}[11]$.

Fig. 1(a) and (b) shows the comparison among model predictions and experimental data of vaporisation rate for water and benzene, respectively. The results from the present model and the A-S '89 model [8] are presented. Two sets of predictions for each model are reported, corresponding to different assumptions for the liquid temperature. The first one corresponds to the results obtained calculating the liquid asymptotic temperature according to Equation (24), the second one imposing the value as reported by Ref. [11], which is about $5 \mathrm{~K}$ and $10 \mathrm{~K}$ higher than the predicted ones for water and benzene drops, respectively. An additional dashed line is plotted, to indicate the perfect agreement between computed and experimental data.

The results show that the differences between the two models are negligible for low evaporation rate (at the order of $10^{-8} \mathrm{~kg} / \mathrm{s}$ ), since the effect related to the non-uniformity of the gas density is expected to be minor. Moreover the right estimation of the liquid temperature is crucial under these conditions, suggesting that both models under-estimate its value and this induces an underprediction of the evaporation rate. This can be explained by noticing that according to the energy conservation Equation (24), the calculation of drop temperature, under steady-state condition, for less volatile liquids with high latent heat of vaporization (like water), results to be crucial, and a small error in its prediction may have a pronounced effect in the calculation of the evaporation rate.

Furthermore, the results enlightened that the difference between the present model and the A-S '89 model increases with the liquid temperature, and consequently with the vaporization rate, suggesting that gas density gradient may play a more significant role in case of higher drop temperature. When the value of the drop temperature has been assumed equal to that reported in Ref. [11], which is always higher than that calculated solving the energy equation, higher vaporization rate are predicted, then the discrepancy between the two models increases.

Fig. 2(a) and (b) shows the corresponding comparison among model predictions and experimental data of the difference between air temperature at free stream and drop temperature, for water and benzene, respectively, under the same conditions of Fig. 1. The values of the temperature difference are in the range of $15 \mathrm{~K}$ and $60 \mathrm{~K}$ for water and of $20 \mathrm{~K}$ and $25 \mathrm{~K}$ for benzene, then at this relatively low gas temperature conditions, a small deviation in the prediction of liquid temperature has a not negligible effect on the prediction of the gas/drop temperature difference, which drives the drop evaporation under quasi steady-state regime.

\subsection{Data base of Downing, 1966 [12]}

The experimental data base of [12] has been used to compare the model predictions with the data of liquid drop temperature and size for benzene and hexane drops evaporating in a nominal $1 \mathrm{~m} / \mathrm{s}$ air stream. The accuracy of drop size measurements was equivalent to $\pm 0.01 \mathrm{~mm}$, as reported in Ref. [12].

Fig. 3 shows the air/drop temperature difference for benzene drops as function of air temperature, as measured by Ref. [12] and calculated by the A-S '89 model [8] and the present model. The results show that the present model is in very good agreement with the experimental measurements, particularly at high air temperature conditions, where the non-uniformity of gas density, explicitly accounted by the present model, is expected to play a major role in drop heating and evaporation.

Fig. 4 presents the temporal evolution of drop size for hexane drop evaporating in air at $437 \mathrm{~K}, 464 \mathrm{~K}$ and $546 \mathrm{~K}$ (again from Ref. [12]). Under these moderate evaporating conditions the difference between the two models is not significant, with both models predicting the evaporation rate with rather good agreement. It's necessary to remark that no detailed modelling of liquid temperature is included in this calculations, since its effect is beyond the scope of the present study.

Since the drop evaporation rate, when the liquid temperature has reached a quasi-steady value, can be estimated by the slope of the curve on the $D^{2}$-time graph, according to the $D^{2}$-law, a linear interpolation of the experimental and computational data has been performed. The results suggest that with both models the discrepancy from the experimental data is always of the same order except under the highest gas temperature conditions, where the discrepancy is about $18 \%$ with the A-S ' 89 model and reduces to $8 \%$ with the present model.
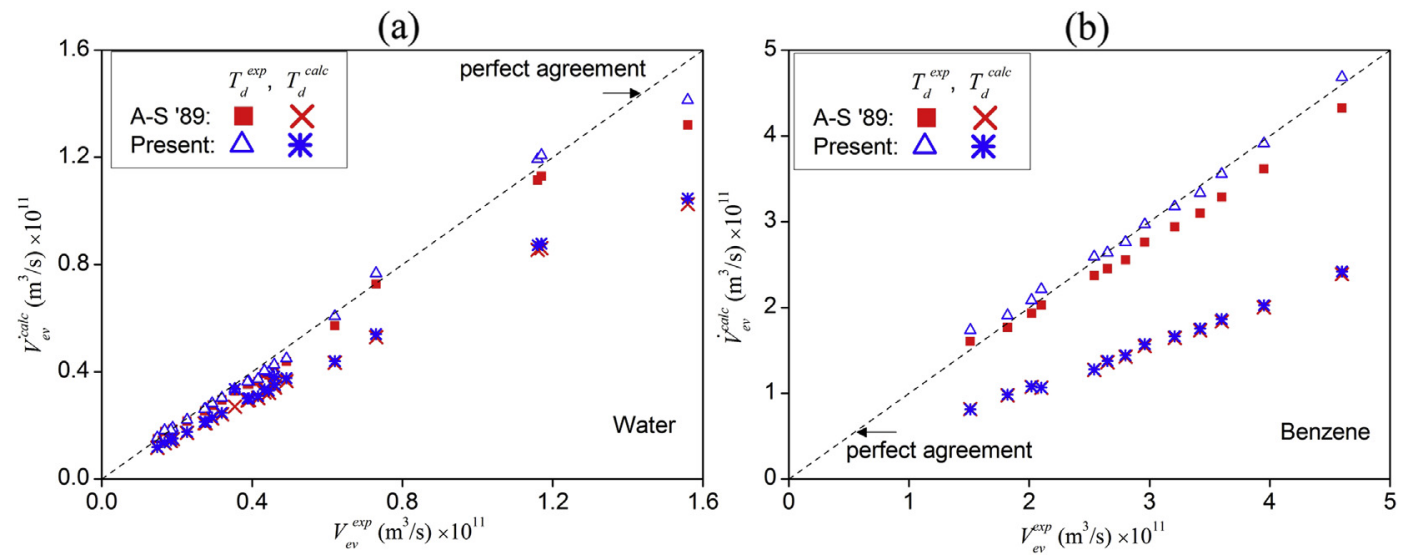

Fig. 1. Comparison among model predictions and experimental measurements [11] of drop volumetric vaporisation rate for (a) water and (b) benzene drops. 
(a)

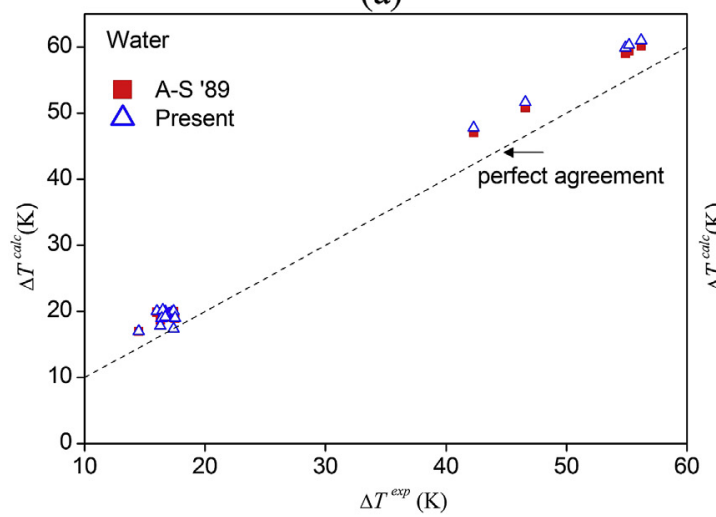

(b)

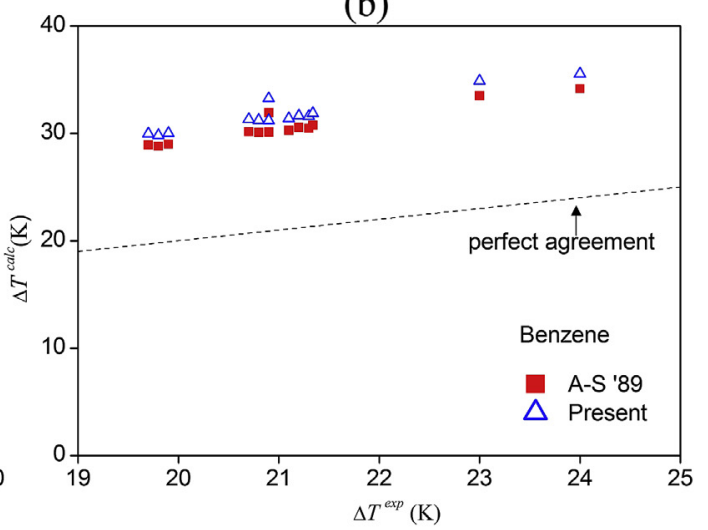

Fig. 2. Comparison among model predictions and experimental measurements [11] of air/drop temperature difference for (a) water and (b) benzene drops.

\subsection{Data base of Eisenklam et al., 1967 [13]}

The evaporation rate of heptane, pentane, methyl alcohol, ethyl alcohol, water and benzene drops, under convective high temperature gaseous flow conditions, was reported by Eisenklam et al., 1966 [13], as function of Reynolds number in terms of a nondimensional evaporation parameter, defined as:

$\Psi=-\frac{\mathrm{d} D^{2}}{\mathrm{~d} t} \frac{\rho_{l} h_{L V}}{4 k_{\mathrm{f}}\left(T_{\infty}-T_{S}\right)}\left(1+B_{\mathrm{f}}\right)$

that is the non-dimensional slope of the curve on the $D^{2}-t$ graph. The tolerance of the parameter $\Psi$ was $\pm 18 \%$ [13].

Three values of gas temperature had been selected equal to $473 \mathrm{~K}, 673 \mathrm{~K}$ and $873 \mathrm{~K}$.

Fig. 5(a) presents the comparison among model predictions and experimental data of the time derivative of drop diameter square for heptane drops. The present model is in good agreement with the experimental data under the whole range of operating conditions, while the A-S '89 model [8] clearly overpredicts the evaporation rate at higher air temperatures. The corresponding values of air/drop temperature difference as predicted by the models and reported in Ref. [13] are shown in Fig. 5(b). Since the temperature difference is well predicted by both models, the better accuracy of the new model in predicting the evaporation rate seems to confirm that explicitly accounting for gas density gradients plays a more relevant role as the evaporation rate increases.

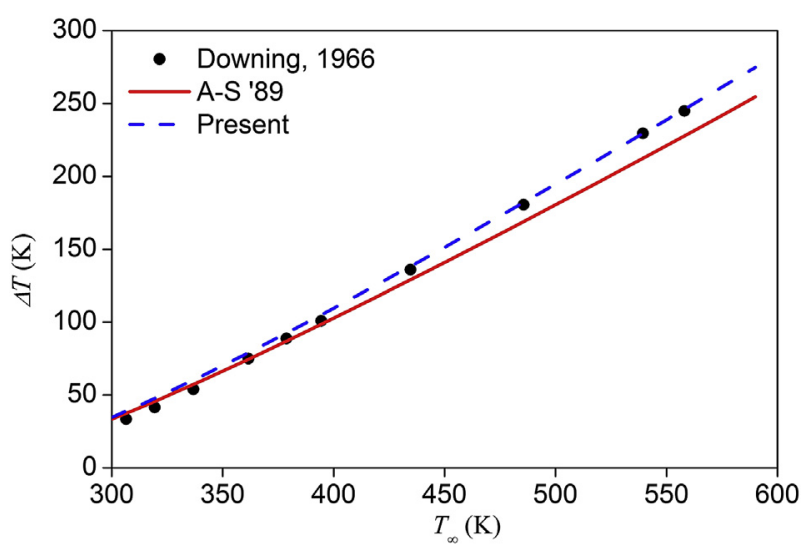

Fig. 3. Comparison among model predictions and experimental measurements [12] of air/drop temperature difference as function of gas temperature for benzene drops.
The experimental data reported in Fig. 2 of [13] are not distinguished according to the air temperature, which had been varied in the range between $473 \mathrm{~K}$ and $873 \mathrm{~K}$. To allow at least a qualitative comparison, it was decided to calculate the parameter $\Psi$ at each selected Reynolds number assuming the air temperature equal to $473 \mathrm{~K}, 673 \mathrm{~K}$ and $873 \mathrm{~K}$. Then for both models, three sets of data predictions are plotted, corresponding to the three air temperatures selected and the band comprised between the predictions at $T_{\infty}=473 \mathrm{~K}$ and $T_{\infty}=873 \mathrm{~K}$ should encompass the experimental data.

The six images of Fig. 6 show the comparison between experimental data and model predictions of the variable $\Psi$ as function of the Reynolds number for pentane, heptane, methyl alcohol, ethyl alcohol, water and benzene drops. The two models calculate the asymptotic temperature according to Equation (24). For all the liquids, the differences between the two models increases with air temperature.

The test-cases with pentane and heptane drops (Fig. 6(a) and (b), respectively) enlighten a good agreement among the predictions from the present model and the experimental data, which values are comprised within the band formed by the simulation results at the two extreme gas temperatures. The A-S '89 model [8] seems to over-estimate the evaporation rate under these operating conditions.

The test-cases with alcohol drops (Fig. 6(c) and (d), respectively) show a relatively higher variation in the vaporisation rate with Reynolds number as recorded by the measurements than predicted by the two models. The results from the present model are in better agreement with the experimental data for Reynolds number lower than about 10 for both species.

The results from the present model are in a relatively good agreement with the experimental data for the case of water drops with Reynolds number lower than 1 , while with higher Reynolds numbers the highest differences recorded in this study are shown in Fig. 6(e), where it is evident that both models underpredict the measured evaporation rate.

The test-case with benzene drops shown in Fig. 6(f) again confirms the relative good agreement among the experimental data and the prediction from the present model, while the classical model predicts higher vaporisation rates for the whole range of operating conditions.

To summarise, the results suggest that an increasing discrepancy between predictions and experiments can be detected as Reynolds number increases for the two alcohols and for water. This can be explained considering that, among the six selected fluids, the two alcohols and water have the highest latent heat of 
(a)

(b)

(c)

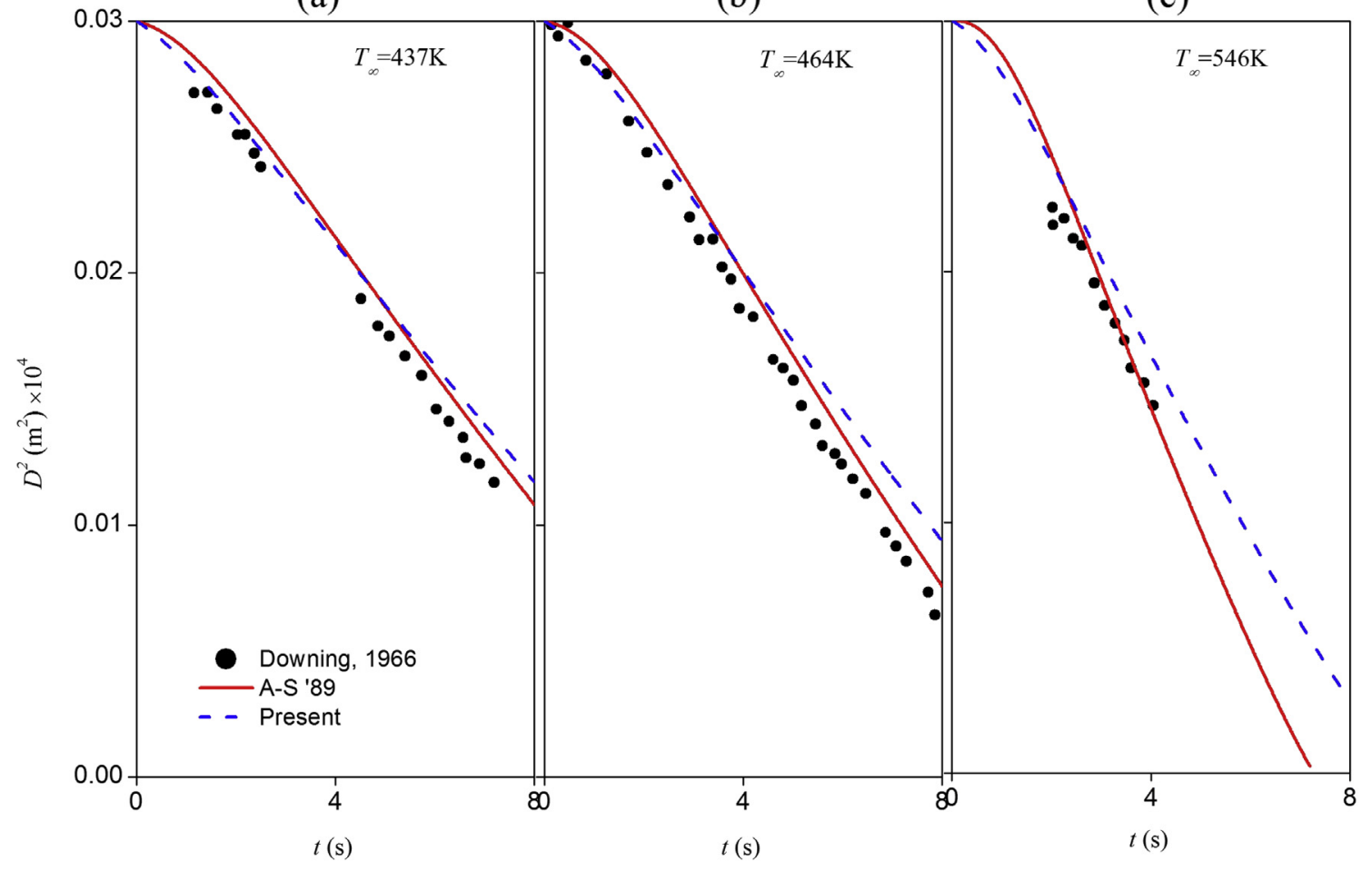

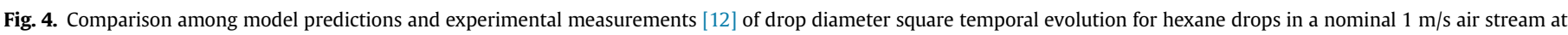
temperature equal to (a) $437 \mathrm{~K}$, (b) $464 \mathrm{~K}$ and (c) $543 \mathrm{~K}$.

vaporization, almost an order of magnitude above the other three fluids (pentane, heptanes and benzene). As previously commented for the test cases of Ranz and Marshall [11], according to the energy conservation Equation (24), the calculation of drop temperature, under steady-state conditions, results to be more crucial in case of fluids with higher latent heat of vaporization, since a small error in its prediction has a more pronounced effect in the calculation of the evaporation rate.

\subsection{Unsteady drop evaporation measurements of Wong and Lin, 1992 [14]}

The last data base of experimental data refers to [14], which measured the temporal evolution of drop size and temperature for a decane drop evaporating in air at $1000 \mathrm{~K}$ and with initial Reynolds number equal to 17 . The drop size was measured with a tolerance of $\pm 2.5 \%$, while the resolution of the temperature acquisition system was $1 \mathrm{~K}$ [14]. Fig. 7 presents the corresponding results, confirming that the effect of variable gas density taken into account by the present model plays a dramatic role under high evaporating conditions. The classical model [8] over-estimates the liquid temperature, which reaches boiling conditions, making the model to diverge.

The present models predict, with a satisfactory agreement, the drop size reduction and the temperature increases. The disagreement between the drop temperature experimental data and the model predictions, which is of the order of few degree along the whole temporal window, seems not to have in this case a significant effect on the correct prediction of the evaporation rate, differently than under low evaporating conditions (see Figs. 1 and 2), since the difference between the gas and drop temperatures is high and then the relative error is lower and of minor importance. As above remarked, no detailed model of liquid temperature distribution inside the drop is included in the present investigation and this (a)

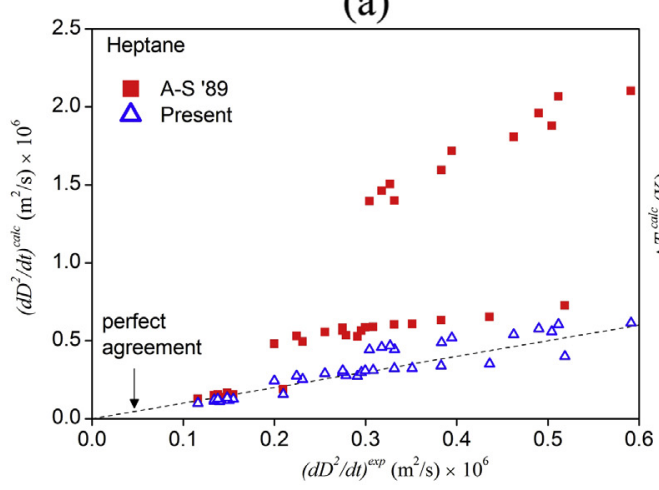

(b)

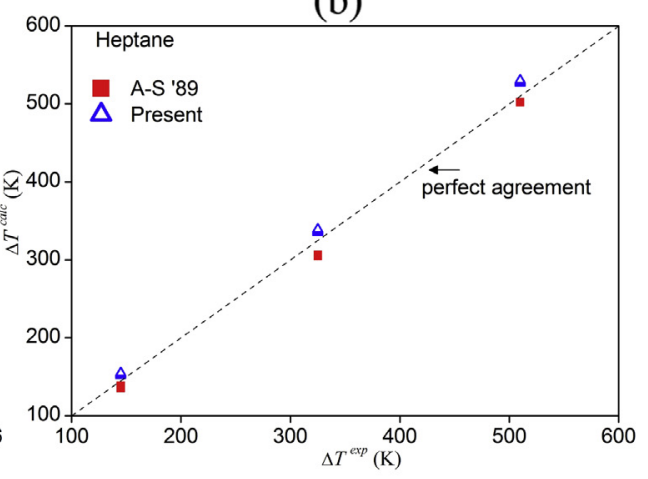

Fig. 5. Comparison among model predictions and experimental measurements [13] of (a) vaporisation rate and (b) air/drop temperature difference for heptane drops. 

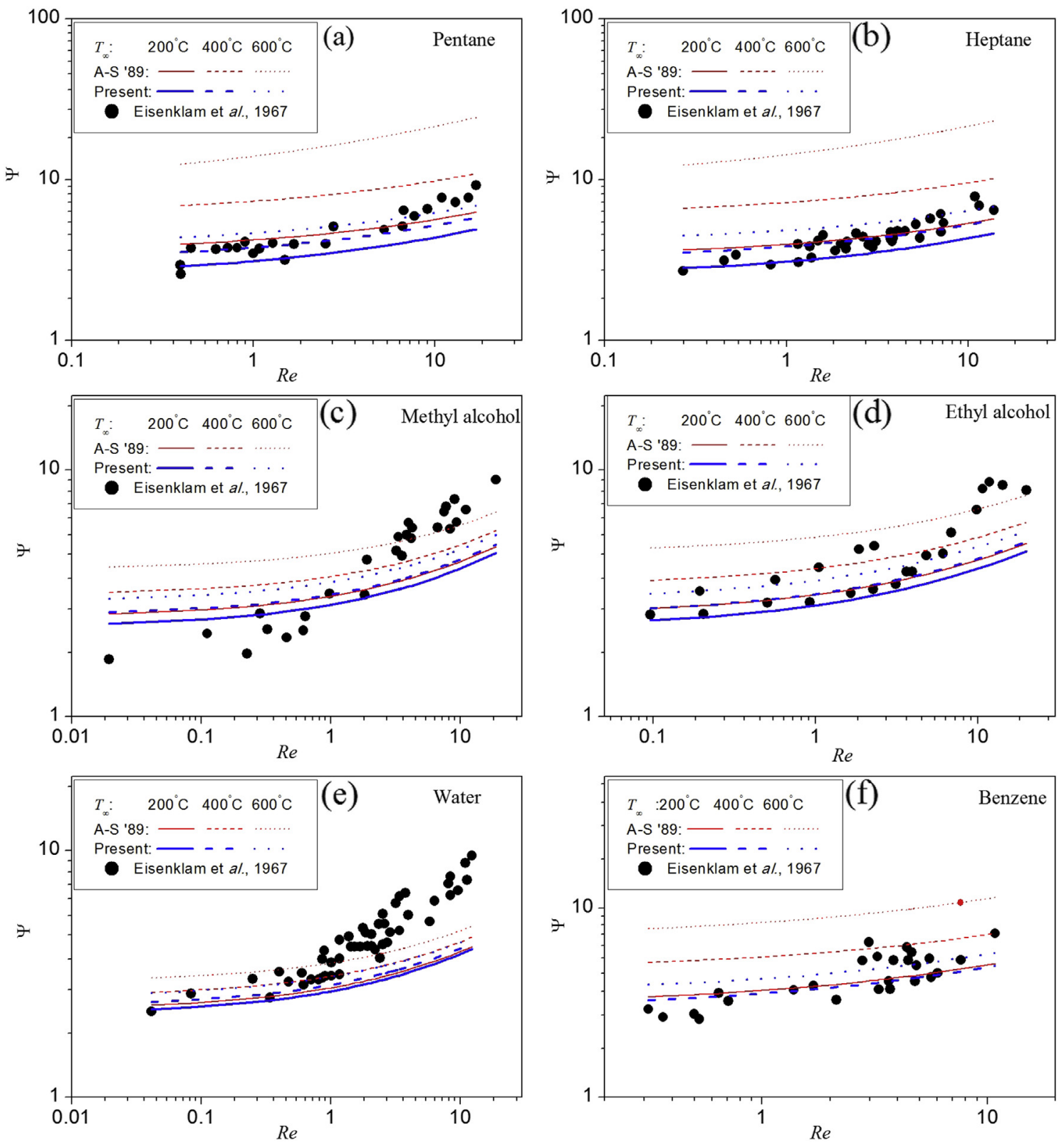

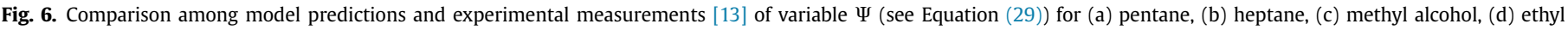
alcohol, (e) water and (f) benzene drops as function of Reynolds number and air temperature.

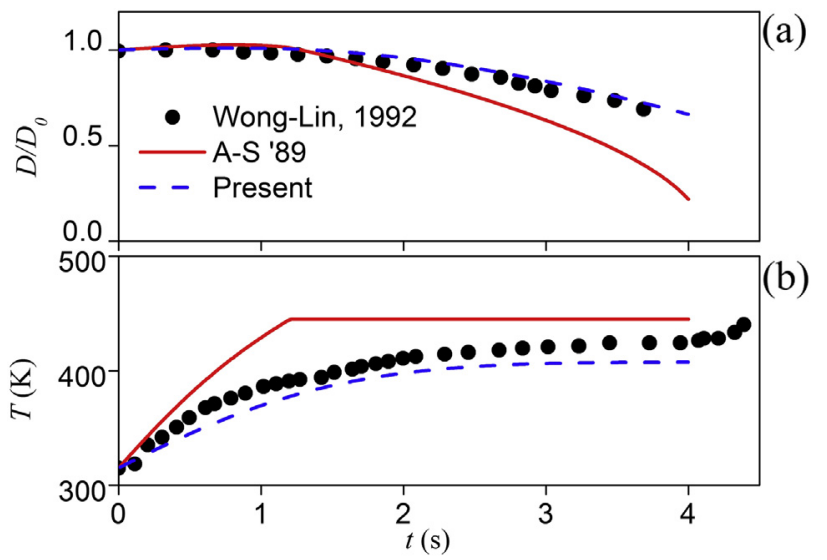

Fig. 7. Comparison among model predictions and experimental measurements [14] of decane drop (a) size and (b) temperature temporal evolution. may explain part of the discrepancies between the model predictions and the experimental data.

These results confirm the satisfactory reliability of the present model to predict the phenomenon of drop evaporation under a wide range of convective conditions. Moreover the analytical solution of the proposed model has a rather simple implementation, making it suitable for comprehensive CFD spray simulations.

\section{Conclusions}

A new model predicting the drop evaporation in hot environment under convective conditions has been developed, based on classical extended film model proposed by Abramzon and Sirignano back in 1989, introducing a thermal and a diffusional thickness for the solution of the heat and mass transfer equations. The model accounts explicitly the dependence of gas density and temperature on specie concentration, relieving the inconsistency of 
commonly used evaporation models and at the same time it can be rather simply implemented in single drop and/or spray CFD codes.

The results from the simulation of quasi steady-state and transient drop evaporation confirm that for this comparison the present model yields good prediction of the experimental data in the whole range of operating conditions investigated. Moreover, at high evaporation rate conditions the present model is able to calculate, with a rather satisfactory accuracy respect to the classical model, the drop size and temperature evolution.

\section{Acknowledgements}

This work was partially financed by the Project V.I.R. Berg. cofinanced by Regione Lombardia.

\section{References}

[1] Z. Zhifua, W. Guoxianga, C. Bina, G. Liejina, W. Yueshea, Evaluation of evaporation models for single moving droplet with a high evaporation rate, Powder Technol. 240 (2013) 95-102.

[2] S.K. Aggarwal, F. Peng, A review of droplet dynamics and vaporization modeling for engineering calculations, J Eng. Gas Turbines Power 117 (3) (1995) 453-461.

[3] G.A.E. Godsave, Studies of the combustion of drops in a fuel spray - the burning of single drops of fuel, in: Fourth Symposium (International) on Combustion, Williams and Wilkins, Baltimore, MD, 1953, pp. 818-830.

[4] D.B. Spalding, The combustion of liquid fuels, in: Fourth Symposium (International) on Combustion, Williams and Wilkins, Baltimore, 1953, pp. $847-$ 864.

[5] R.S. Miller, K. Harstad, J. Bellan, Evaluation of equilibrium and non-equilibrium evaporation models for many-droplet gas-liquid flow simulations, Int. J. Multiphase Flow 24 (1998) 1025-1055.
[6] S. Sazhin, Advanced models of fuel droplet heating and evaporation, Prog. Energy Combust. Sci. 32 (2006) 162-214.

[7] W.A. Sirignano, Fluid Dynamics and Transport of Droplets and Sprays, second ed., Cambridge University Press, 2010.

8] B. Abramzon, W.A. Sirignano, Droplet vaporization model for spray combustion calculations, Int. J. Heat Mass Transf. 32 (9) (1989) 1605-1618.

[9] M. Renksizbulut, M.C. Yuen, Experimental study of droplet evaporation in a high temperature air stream, J. Heat Transf. 105 (1983) 384-388.

[10] Ranz, Marshall, Evaporation from drops, part I, Chem. Eng. Prog. 48 (4) (1952) $141-146$.

[11] Ranz, Marshall, Evaporation from drops, part II, Chem. Eng. Prog. 48 (4) (1952) $173-180$.

[12] C.G. Downing, The evaporation of drops of pure liquids at elevated temperatures: rates of evaporation and wet-bulb temperatures, AIChE J. 12 (4) (1966) $760-766$.

[13] P. Eisenklam, S.A. Arunachalam, J.A. Weston, Evaporation rates and drag resistance of burning drops, in: The Eleventh Symposium on Combustion, The Combustion Institute, Pittsburgh, P.A., 1967, pp. 715-728.

[14] S.C. Wong, A.C. Lin, Internal temperature distributions of droplets vaporizing in high-temperature convective flows, J. Fluid Mech. 237 (1992) 671-687.

[15] M.C. Yuen, L.W. Chen, On drag of evaporating droplets, Combust. Sci. Tech. 14 (1976) 147-154.

[16] M.C. Yuen, L.W. Chen, Heat transfer measurements of evaporating liquid droplets, Int. J. Heat Mass Transf. 21 (1978) 537-542.

[17] S. Tonini, G.E. Cossali, An analytical model of liquid drop evaporation in gaseous environment, Int. J. Therm. Sci. 57 (2012) 45-53.

[18] S. Bird, Transport Phenomena, Lightfoot, 2002.

[19] R. Clift, J.R. Grace, M.E. Weber, Bubbles, Drops, and Particles, Academic, New York, 1978.

[20] N.A. Fuchs, Vaporisation and Droplet Growth in Gaseous Media, Pergamon Press, London, 1959.

[21] B. Abramzon, S. Sazhin, Droplet vaporization model in the presence of thermal radiation, Int. J. Heat Mass Transf. 48 (2005) 1868-1873.

[22] S. Tonini, Heat and mass transfer modelling of sub-micrometer droplets under atmospheric pressure conditions, Atomization Sprays 19 (9) (2009) 833-846. 